\title{
Adolfo Longhitano
}

\section{Il gruppo italiano docenti di diritto canonico origine, scelte metodologiche, attività}

Prawo Kanoniczne : kwartalnik prawno-historyczny 43/3-4, 323-349

2000

Artykuł został zdigitalizowany i opracowany do udostępnienia w internecie przez Muzeum Historii Polski w ramach prac podejmowanych na rzecz zapewnienia otwartego, powszechnego i trwałego dostępu do polskiego dorobku naukowego i kulturalnego. Artykuł jest umieszczony w kolekcji cyfrowej bazhum.muzhp.pl, gromadzącej zawartość polskich czasopism humanistycznych i społecznych.

Tekst jest udostępniony do wykorzystania w ramach dozwolonego użytku. 
Prawo Kanoniczne

43 (2000) nr 3-4

\section{IL GRUPPO ITALIANO DOCENTI DI DIRITTO CANONICO ORIGINE, SCELTE METODOLOGICHE, ATTIVITÀ}

\section{Dalle prime riunioni ai convegni annuali: la nascita del Gruppo}

Sono note le difficoltà incontrate dai docenti di diritto canonico dopo la chiusura del Vaticano $\mathrm{II}^{1}$ : alcuni non riuscivano a svolgere serenamente il consueto programma, non solo perché non erano più in grado di stabilire quali norme del codice pio-benedettino erano ancora in vigore e quali erano già state abrogate, ma soprattutto per la contestazione che si era manifestata in diversi seminari o studentati teologici: a volte gli alunni avevano apertamente contestato il professore di diritto canonico o si erano anche allontanati dall'aula al suo ingresso;

\footnotetext{
* Ordinario di diritto canonico nello Studio Teologico San Paolo di Catania (Facoltà teologica della Sicilia).

Questo saggio rielabora la relazione tenuta al XXVII incontro di studio del Gruppo italiano docenti di diritto canonico, tenutosi a Borca di Cadore nei giorni 26-30 giugno 2000.

'Un atteggiamento antigiuridico si era manifestato nello svolgimento del Concilio Vaticano II, soprattutto durante il dibattito sulla costituzione della Chiesa. Il termine giuridismo (o giuridicismo), se non fu coniato in concilio ( $\mathrm{fu}$ certamente adoperato dal patriarca melchita Maximos IV), divenne di uso comune al punto da meritare un richiamo specifico nell'indice analitico del più noto commento alla Lumen Gentium: La Chiesa del Vaticano II, a cura di G. Baraúna, Firenze 1966. In questo stesso volume Y. Congar, auspicando e prospettando con estrema lucidità una nuova visione del diritto canonico, così scriveva nelle conclusioni: «Si rifletterà di nuovo, non soltanto sulla natura e il luogo del diritto nella Chiesa ma sulle fonti dell'obbligo. Si vedrà che non esistono soltanto precetti fondati in una legge in senso strettamente giuridico, coattiva, ma anche obblighi derivanti dalla comunione, e dunque, finalmente, dall'amore. Si metterà in auge un diritto di comunione. Si tornerà a questa nozione, chiave di tutta la Chiesa antica e si riscriverà l'ecclesiologia in questa luce. Non si dovrà più procedere negativamente nella necessaria critica del giuridicismo: che invece partirà dai grandi valori, totalmente positivi, di servizio, missione, comunione, culto della fede...» (ivi, 1267). La prospettiva enunciata dal Congar si sarebbe realizzata a distanza di anni, dopo un periodo di incertezze, incomprensioni e vivaci dibattiti.
} 
nella maggior parte dei casi si mostravano insofferenti o disinteressati alle lezioni.

C'era in tutti il desiderio di confronto per discutere, per conoscere le esperienze degli altri e per sottoporre le proprie al loro giudizio. La mia esperienza di docente era simile a quella dei colleghi incontrati in quegli anni. Incominciai ad insegnare nel primo anno di vita dello Studio Teologico San Paolo di Catania (1969-1970); per consentire agli studenti provenienti da seminari diversi di completare il programma, fu necessario che $\mathrm{i}$ tre docenti di diritto canonico assicurassero contemporaneamente tutti i corsi previsti dalla ratio studiorum: $\mathrm{mi}$ trovai ad improvvisare il corso introduttivo e l'insegnamento del libro IV sui processi e del libro V sui delitti e le pene.

L'unica possibilità di stabilire contatti con gli altri docenti era la partecipazione ai convegni che si tenevano annualmente in Italia. Nel 1970 presi parte al Congresso canonistico-pastorale indetto da mons. Fiorenzo Romita, promotore dell'Associazione canonistica italiana; nel 1971 intervenni al congresso internazionale di Roma, dove ottenni dal p. Jean Beyer di poter partecipare al Colloquium iuris canonici, organizzato dalla Pontificia Università Gregoriana per i suoi ex alunni.

La decisione di incontrarsi per dar vita a iniziative concrete fra i docenti di diritto canonico dei seminari e degli studentati religiosi fu presa a Napoli, in occasione del II Congresso canonistico-pastorale del 1970. L'interesse principale non era costituito dalla particolare concezione del diritto, che mons. Romita cercava di far passare nelle relazioni da lui commissionate e controllate puntigliosamente prima di essere pubblicate nel Monitor Ecclesiasticus, ma dall'occasione di un incontro per discutere e dibattere i propri problemi.

Fra i primi docenti che si dissero disposti a far conoscere le proprie esperienze ricordo Aldo Bressani - che presentò agli altri Agostino Martini e le sue pubblicazioni in tema di antropologia giuridica e di teologia del diritto - Attilio Giacobbi, Nino Minetti, Agostino Vallini, Giovanni Ricci, Annetto De Pasquale, i salesiani Gustave Leclerc e Pier Giorgio Marcuzzi.

Fu questo il primo nucleo del Gruppo che decise di dar vita alle seguenti iniziative: inviare a don Minetti (che diede la propria disponibilità a fare da segretario): a) la ratio studiorum di tutto il corso teologico dei vari seminari, o almeno del diritto canonico con i necessari riferimenti, indicando il numero di ore e il programma dettagliato per 
ogni anno; b) un ampio schema dei trattati già svolti con la bibliografia ragionata; c) eventuali dispense da mettere a disposizione di tutti.

L'appuntamento per l'anno successivo fu sempre a Napoli al III Congresso canonistico-pastorale (13-17 settembre 1971). I docenti avevano già in mano gli schemi e le dispense messe a disposizione da Martini, Leclerc e Longhitano e la ratio studiorum di alcuni seminari (Savona, Belluno, Udine, Catania, Napoli, Francescani di Verona...).

Per prendere in esame questo materiale, un gruppo ristretto di docenti si riunì nel mese di aprile del 1972 a Roma, ospiti di don Leclerc al Pontificio Ateneo Salesiano. Da questo primo esame si incominciarono a delineare due metodologie diverse, che furono sintetizzate ed esposte in due schemi: uno innovativo redatto da Martini e uno tradizionale preparato da Marcuzzi. Di questo incontro a Roma abbiamo un'ampia relazione su Salesianum ${ }^{2}$. Il relatore, dopo aver esposto le difficoltà che incontravano i docenti nel dovere insegnare un codice in gran parte superato dal Concilio Vaticano II, non mancò di manifestare la propria preoccupazione per le proposte innovative avanzate da alcuni: se il corso di diritto canonič assumeva il nome di «Ecclesiologia istituzionale» ed era sviluppato sullo schema della Lumen Gentium, era fondato il timore che il diritto potesse essere assorbito dalla teologia e perdere la sua specificità.

I due schemi furono spediti a tutto il gruppo con l'invito ad ognuno di preparare le osservazioni da presentare al IV Congresso canonistico-pastorale di Bologna (25-29 settembre 1972). I rilievi fatti dai docenti evidenziarono la scelta per lo schema innovativo, che aveva una chiara e coerente logica interna. Allo schema tradizionale si rimproverava la mancanza di coerenza sui temi fondamentali: il diritto, la Chiesa, il rapporto fra comunità ecclesiale e comunità politica... In poche parole: si aveva l'impressione che si volesse mantenere intatto l'insegnamento tradizionale, limitandosi a qualche citazione superficiale del Vaticano II.

Fu in questo clima che il Gruppo docenti decise di indire un convegno annuale al fine di approfondire una concezione del diritto canonico più rispondente ai dati del Vaticano II e di predisporre gli strumenti idonei all'insegnamento. Nel gennaio successivo si tenne una riu-

${ }^{2} \mathrm{G}$. Leclerc, L'insegnamento del diritto canonico. Situazione e problemi, in Salesianum 33 (1972) 315-319. 
nione ristretta al Seminario Guanelliano di Roma, ospiti di don Minetti, dove si decise di convocare un convegno per la prima settimana di luglio del 1973, al passo della Mendola (Villa Toval). Il segretario del gruppo fece stampare il programma, che spedì ai docenti di diritto canonico dei seminari d'Italia ${ }^{3}$. La celebrazione del primo convegno segnò la nascita del Gruppo. I docenti promotori, chiusa la fase preparatoria, si ritrovarono uniti e decisi ad organizzarsi e a fornirsi degli strumenti idonei per raggiungere il fine prefisso ${ }^{4}$.

L'incontro della Mendola fu articolato su due relazioni di fondo. La prima, tenuta da don Gustave Leclerc, aveva come titolo: Panoramica

\footnotetext{
${ }^{3}$ Non partecipai a questo primo convegno per il sopravvenire di impegni di diversa natura. Da una cartolina di saluti che mi fu inviata leggo fra i partecipanti i nomi di Agostino Martini, Umberto Betti, Attilio Giacobbi, Mosè Francescato, Aldo Bressani, Francesco Cavarra, Gustave Leclerc, Pier Giorgio Marcuzzi, Giovanni Ricci, Alberto Tanasini, Giancarlo Manzoli, Agostino Vallini, Bruno Senofonte, Nino Minetti, Guido Carretto...

' Il Gruppo non si preoccupò di redigere uno statuto e di formalizzare l'iscrizione dei membri. L'organizzazione si sviluppò negli anni a partire da esigenze concrete, in uno spirito di servizio e nella logica della condivisione/comunione: per raggiungere i docenti dei seminari e degli istituti religiosi si pensò di istituire gli incaricati regionali (a qualcuno furono assegnate più regioni); per dare ai membri la possibilità di conoscersi e di mantenere i contatti si iniziò a stampare l'Elenco docenti, aggiornato periodicamente; al segretario si affiancarono altri due docenti, che insieme costituirono la segreteria nazionale. II segretario aveva il compito di coordinare le attività e di informare il Gruppo: almeno una volta l'anno inviava una circolare con le „notizie di famiglia", contattava i relatori dei convegni annuali, provvedeva a inviare ai membri il programma del convegno. Nel corso degli anni nella segreteria nazionale furono cooptati altri docenti con incarichi generali (rapporti con Roma, bibliografia canonistica, amministrazione, rapporti con il CATI). A partire dal 1976 la segreteria nazionale e i segretari regionali incominciarono a riunirsi a Roma nel mese di novembre per preparare il convegno dell'estate successiva. Normalmente le proposte erano discusse nelle riunioni della segretaria nazionale e dei segretari regionali (temi dei convegni, relatori, rapporti con l'esterno...); decideva l'assemblea dei docenti durante il convegno annuale. Per le spese di segreteria e per l'organizzazione dei convegni il Gruppo si è sempre autofinanziato. L'ufficio di segretario nazionale fino al 1977 fu retto da don Nino Minetti; gli subentrò una terna composta da don Attilio Giacobbi (segretario), don Tarcisio Bertone e p. Agostino Montan. Nel 1981 si ebbe un ricambio e la terna fu composta dal p. Agostino Montan (segretario), don Cesare Gentili e don Adolfo Longhitano. Nel 1987, dopo le dimissioni del p. Montan, entrò a far parte della segreteria il p. Velasio De Paolis; l'ufficio di segretario nazionale fu assunto da don Cesare Gentili, che si avvalse della collaborazione della signora Anna Menconi. Nel 1994 si aggiunsero alla segreteria nazionale il p. Vincenzo Mosca (economo) e don Carlo Redaelli (responsabile della bibliografia canonistica a schede); nel 1997 fu cooptato don Adriano Celeghin (responsabile dei contatti con il CATI). Nel 1998 nell'ufficio di segretario nazionale Mons. Cesare Gentili fu sostituito da Mons. Gian Paolo Montini.
} 
centrale della situazione dell'insegnamento del Diritto Canonico nel concerto delle varie discipline teologiche e nell'ambito della nuova «Ratio studiorum». La seconda, tenuta dal p. Agostino Martini, si proponeva di delineare una Introduzione al Diritto Canonico.

\section{Le scelte metodologiche}

Per comprendere il metodo di lavoro, che il primo nucleo del Gruppo intendeva seguire per raggiungere le proprie finalità, possiamo leggere il brano centrale della lettera con cui il segretario don Nino Minetti accompagnava il programma:

«Egregio professore, Le scrivo per metterLa a conoscenza di una iniziativa che la sezione „Docenti di Diritto Canonico” dell'Associazione Canonistica Italiana vuol promuovere e a cui si vorrebbe assicurare per tempo la Sua adesione. L'iniziativa è nata dalla collaborazione che in questi ultimi tre anni si è venuta sviluppando tra i Docenti in occasione dei Congressi dell'Associazione. Oltre infatti allo scambio di idee sull'insegnamento e alla comunicazione di dispense e pubblicazioni già attuata finora, si è sentita urgente la necessità di giungere alla realizzazione di uno strumento didattico concreto: fissare le linee di un nuovo programma di insegnamento di diritto canonico nei seminari maggiori e negli studentati teologici e fornire i sussidi. Si è pensato quindi di realizzare questo piano di lavoro: a) tramite convegni di studio, b) a larga partecipazione di docenti per avere l'apporto costruttivo di una grande varietà di esperienze e di persone e per dare al lavoro un'impronta qualificata; c) con scopo eminentemente pratico: giungere alla costruzione di quaderni-guida, comprensivi di tutto il ciclo istituzionale, anche da pubblicare; d) attraverso un lavoro attivo di tutti: dopo la presentazione del tema da studiare, con una o al massimo due relazioni orientative, dare largo spazio ai lavori di gruppo; questi dovranno stabilire quali contenuti dare alle varie parti del programma e come darli, in più dovranno arrivare a svilupparne, per quanto possibile, una traccia scritta. Le assemblee avranno il compito di discutere e coordinare gli elaborati di gruppo. Si pensa di affidare la stesura definitiva dei sussidi ad una ristretta équipe di Docenti».

Il primo convegno intendeva affrontare il problema del programma: come articolare i corsi tenendo conto delle nuove direttive conciliari? Le due tendenze emerse nelle prime riunioni (quella innovativa e quella tradizionale) si contrapposero in modo netto quando si trattò di formulare lo schema del programma di diritto canonico da inserire 
nella ratio studiorum. Il p. Agostino Martini proponeva che il diritto canonico fosse sviluppato in stretta connessione con i principi teologici fatti propri dal concilio; il salesiano don Gustave Leclerc rifiutava questa proposta, perché temeva che il canonista si trasformasse in teologo e si finisse col far perdere al diritto canonico la sua identità (in un suo articolo pubblicato nello stesso periodo in Salesianum si capì che egli identificava il diritto con la norma positiva, secondo l'indirizzo della scuola canonistica italiana $)^{5}$. Fu chiaro a quel punto che c'era da fare una scelta di principio e di metodo. Quando sembrava inevitabile una rottura, si profilò un compromesso: elaborare due schemi diversi, secondo le due tendenze emerse fra i docenti del Gruppo.

\subsection{Le indicazioni del Vaticano II e la ricerca di un nuovo metodo di ricerca e di insegnamento}

Il punto di partenza in cui tutti dicevano di ritrovarsi era l'indicazione contenuta nella Optatam Totius 16: "Nella esposizione del diritto canonico [...] si tenga presente il mistero della Chiesa, secondo la costituzione dogmatica De Ecclesia». Il problema era di individuare il percorso più idoneo per accogliere questa indicazione e tradurla in una trattazione organica, coerente e condivisa. Alla luce di queste parole, alcuni ritenevano del tutto superate, e quindi inutilizzabili per l'insegnamento, le tesi sostenute nel periodo preconciliare dallo Ius publicum ecclesiasticum, sia pure nella visione aggiornata della scuola canonistica italiana ${ }^{6}$; altri ritenevano possibile, se non doveroso, riproporre il vecchio insegnamento con qualche modifica terminologica: si poteva accettare la visione teologica di una Chiesa definita sacramento di salvezza e continuare a sostenere che la sua giuridicità derivasse dalla inaequalitas dei suoi membri: i chierici, che esercitavano la potestas conferita dall'ordine sacro e i laici, che erano loro sottomessi ${ }^{7}$.

I docenti che si riproponevano di formulare una nuova ipotesi di fondazione del diritto canonico si accorsero che le proposte fatte dalle diverse scuole o ponevano qualche difficoltà dal punto di vista meto-

${ }^{5} \mathrm{G}$. Leclerc, Configurazione della norma canonica nella prospettiva del Concilio Vaticano II, in Salesianum 35 (1974) 615-640.

"V. Del Giudice, Nozioni di diritto canonico, Milano 1962"; P.A. d'Avack, Chiesa cattolica (diritto canonico), in Enciclopedia del Diritto, VI, Milano 1960, 926-942.

${ }^{7}$ Può essere considerata emblematica di questo indirizzo la nuova edizione del manuale di Vincenzo Del Giudice in uso nelle università statali e in alcuni seminari. II curatore Gaetano Catalano asseriva di averlo aggiornato ai dati del concilio. 
dologico o non erano ancora così chiare ed elaborate da essere proposte per l'insegnamento.

1) la soluzione formulata da Wilhelm Bertrams nei suoi corsi alla Gregoriana dal punto di vista metodologico veniva posta sullo stesso piano di quella dello Ius publicum ecclesiasticum, perché non era dedotta da una premessa teologica, ma dalla filosofia sociale del Gundlach;

2) suscitava un certo interesse l'ipotesi formulata da Klaus Mörsdorf e divulgata da Eugenio Corecco e da Antonio Rouco Varela ${ }^{9}$. Tuttavia se si poteva condividere l'analisi critica delle ipotesi di fondazione del diritto canonico del passato, non si riusciva a comprendere la nuova proposta che intendevano illustrare. Infatti, volendo essere estremamente rigorosi nella scelta di un metodo teologico per la fondazione del diritto canonico, evitavano perfino di esplicitare la nozione di diritto alla quale intendevano far riferimento nella loro trattazione, creando ai docenti non poche difficoltà nello sforzo di offrire agli alunni nozioni semplici, chiare e di facile apprendimento;

3) creava una comprensibile perplessità il progetto di deteologizzazione del diritto canonico, proposto dalla rivista Concilium e dai canonisti dell'Istituto di Scienze Religiose di Bologna, perché sembrava percorrere la strada inversa indicata dal Vaticano II, anche se le loro conclusioni non erano prive di interesse ${ }^{10}$.

I convegni annuali alla Mendola, più che per i sussidi offerti, si rivelarono utili per la conoscenza dei docenti di diritto canonico, per l'acquisizione di un metodo di studio e per la formazione dei partecipanti. A tutti si spedivano per tempo gli inviti ai convegni e le circolari con il resoconto dell'attività del Gruppo, nella speranza di coinvolgerli. Coloro che partecipavano per la prima volta al convegno avvertivano di trovarsi a proprio

${ }^{8}$ W. Bertrams, Quaestiones fundamentales iuris canonici, Roma 1969. Per una lettura critica di questa ipotesi e della scuola dello lus publicum ecclesiasticum si veda E. Corecco, Il rinnovo metodologico del diritto canonico, in La Scuola Cattolica 94 (1966) 3-35; Id., Teologia del diritto canonico, in Nuovo dizionario di teologia, a cura di G. Barbaglio e S. Dianich, Alba 1977, 1711-1753.

${ }^{9} \mathrm{~K}$. Mörsdorf, Diritto canonico, in Dizionario teologico, a cura di H. Fries, I, Brescia 1966, 479-490; E. Corecco, Teologia del diritto canonico, cit.; A. Rouco Varela, Le statut ontologique et épistemologique du droit canonique, in Revue des Sciences Philosophiques et Théologiques 57 (1973) 203-227.

${ }^{10} \mathrm{Si}$ veda l'editoriale di Concilium 1 (1965) 8, 1-6 (edizione integrale spagnola); L. Martini - A. Ippoliti, Il dibattito sui fondamenti teologici del diritto canonico, in Legge e Vangelo. Discussione su una legge fondamentale per la Chiesa, Brescia $1972,265-340$. 
agio in un ambiente familiare. Il tema del convegno era introdotto da una relazione teologica, che esponeva i principi dottrinali nella visione data dal Vaticano II per essere poi sviluppato dal punto di vista canonistico. Alle relazioni seguiva la discussione finalizzata al chiarimento e all'approfondimento delle tematiche. Dopo la discussione si preparavano gli schemi provvisori per l'insegnamento, tenendo conto dell'apporto dato nella discussione. Lo schema provvisorio diventava definitivo quando si raggiungeva una sufficiente convergenza sulla formulazione proposta.

Il criterio metodologico seguito dal Gruppo ebbe una prima autorevole conferma negli orientamenti della Santa Sede per l'insegnamento del diritto canonico nelle facoltà teologiche e nei seminari. La Congregazione per l'educazione cattolica, nella lettera circolare del 2 aprile 1975 Insegnamento del diritto canonico per gli aspiranti al sacerdozio (EV 5/1221-1242), faceva notare che negli ultimi anni lo studio del diritto canonico aveva subito, per diverse cause, una perdita di interesse, specialmente tra gli studenti ecclesiastici, con un certo disorientamento nella Chiesa. Questa perdita di interesse veniva attribuita a due difficoltà: «una supposta desuetudine del codice di diritto canonico e mancanza di un codice nuovo; una imperfetta e talora falsa interpretazione dell'ecclesiologia del Concilio Vaticano II» (ivi, 1222). Una risposta alla prima difficoltà non comportava particolari problemi: il codice piobenedettino non era stato ancora formalmente abrogato. Più complessa quella alla seconda difficoltà; ma la Congregazione cercò di dimostrare «come sia proprio l'ecclesiologia del Vaticano II a sollecitare la promozione del diritto nella chiesa» (l.c.) e non fece mancare riflessioni dottrinali e indicazioni operative per i docenti, fra le quali si legge:

«Nell'insegnamento vengano indicati i fondamenti teologici generali del diritto canonico e quelli particolari di ogni istituto giuridico. In tal modo ed in tale linea, sarà messo in evidenza lo spirito che anima il diritto della chiesa, a differenza degli altri diritti, e la sua funzione pastorale» (ivi, 1234).

In quello stesso periodo si preparava la bozza della ratio studiorum per le facoltà teologiche e i seminari. Il Gruppo diede il proprio contributo alla sua elaborazione, com'è documentato in un saggio pubblicato nel 1978 da Agostino Vallini, che riportava in appendice gli schemi dei vari corsi elaborati fino a quel momento ${ }^{11}$.

"A. Vallini, L'insegnamento del diritto canonico nella formazione al sacerdozio, in Monitor Ecclesiasticus 103 (1978) 336-363. 


\subsection{La sfida del manuale «Il diritto nel mistero della Chiesa»}

I risultati ottenuti e le indicazioni della Congregazione per l'educazione cattolica ci indussero a proseguire nel cammino intrapreso, progettando la pubblicazione di un manuale; un obiettivo che all'inizio veniva considerato utopistico. Nel primo volume del nostro manuale, pubblicato nel $1979^{12}$, è documentata la diversa posizione dei membri del Gruppo e lo sforzo di formulare due ipotesi autonome di fondazione del diritto nella realtà umana e nella realtà ecclesiale.

La fondazione del diritto nella realtà umana fu trattata da Agostino Martini. Il suo saggio aveva un indirizzo innovativo per superare i due schemi consueti della filosofia del diritto e della teoria generale del diritto $^{13}$. La scelta non era dettata da un semplice desiderio di novità, ma dalla necessità di trovare vie alternative ai metodi tradizionali di insegnamento del diritto canonico, che l'atteggiamento antigiuridico di quegli anni aveva reso improponibili. L'unica via da percorrere sembrava quella di fondare il diritto sui grandi temi dell'antropologia e della teologia.

L'invito del Concilio Vaticano II $\widetilde{\mathrm{di}}$ comprendere e spiegare il diritto canonico a partire dal mistero della Chiesa poteva essere esteso per analogia al diritto in genere: comprenderlo e spiegarlo a partire da una concezione personalistica dell'uomo e della società. Furono queste le motivazioni che indussero Martini a concepire l'introduzione al diritto nella realtà umana come antropologia giuridica, una disciplina che nella sua elaborazione cercava di coniugare i princìpi del personalismo cristiano con le tesi più aggiornate della filosofia del diritto, sostenute e divulgate da giuristi di matrice cattolica come Francesco Carnelutti, Giuseppe Capograssi e Guido Gonella.

La trattazione del tema nella prima edizione del nostro manuale era più sintetica di quella elaborata nella seconda edizione e seguiva questo schema:

«Premessa. I. I presupposti del progetto giuridico: 1) il progetto uomo; 2) il progetto storico; 3) il progetto socio-politico. II. La specificità del progetto giuridico: 1) Il progetto giudico e la sua imprescindibilità storica; 2) Il progetto giuridico e la sua identità; 3) il progetto giudico e il suo spazio vitale; 4) il progetto giuridico e le sue funzioni

${ }^{12}$ Il diritto nel mistero della Chiesa, Roma 1979.

${ }^{13} \mathrm{~A}$. Martini, ll diritto nella realtà umana, ivi, 1-38. 
promozionali; 5) il progetto giuridico e la sua elaborazione; 6) il progetto giuridico e la sua attuazione. Conclusione».

Se ad una prima lettura si aveva l'impressione di una trattazione del tutto nuova, un esame più attento del testo faceva constatare che erano nuovi lo schema e il linguaggio, in realtà al suo interno venivano recuperate tutte le tematiche tradizionali: la nozione di diritto e le ipotesi formulate dalle diverse scuole, il suo rapporto con la morale, la distinzione fra diritto naturale e diritto positivo, la formulazione del diritto positivo, le funzioni proprie della norma positiva, i problemi della sua interpretazione e della sua attuazione...

Cerchiamo di evidenziare gli elementi di novità contenuti nella trattazione. L'abbandono della filosofia del diritto e della teoria generale del diritto per la scelta dell'antropologia giuridica dava la possibilità di considerare il diritto non più come elemento astratto, quasi estraneo, che solo in un secondo momento si poneva alla coscienza dell'uomo come norma vincolante. Il diritto diventava elemento integrante della persona umana, considerata a sua volta non in modo statico, ma dinamico che deve realizzarsi nella società e nella storia.

Martini, invece di parlare di diritto, preferisce parlare di progetto giuridico, che risulta dalla convergenza di tre progetti: il progetto uomo, il progetto storico, il progetto socio-politico. In questa visione il progetto giuridico poteva essere definito come «convivenza umana strutturata imperativamente dalla comunità politica, in un tutto organico, finalizzata all'ottenimento del bene comune per garantire e promuovere la crescita integrale della persona umana $\gg^{14}$. Una definizione molto ricca che distingueva il diritto dalla norma, assicurava il riferimento a un „quadro di valori", filtrato dalle mediazioni culturali e socio-politiche, evitando in tal modo le concezioni riduzionistiche del diritto: dal normativismo, al giusnaturalismo illuministico, al realismo giuridico. L'autore sottolineava che il suo progetto giuridico non poteva essere concepito come «normatizzazione della vita sociale»una espressione che tradiva le sue matrici positivistiche -, ma come "vita sociale normativata», che permetteva il recupero della migliore tradizione cristiana.

A questi rilievi positivi possiamo aggiungere alcune osservazioni critiche: il ricorso ad un linguaggio ricercato, quasi per iniziati; il ca-

${ }^{14} \mathrm{Ivi}, 16$. 
rattere troppo innovativo dello schema, che non rendeva di facile intuizione il recupero degli elementi considerati fondamentali nei manuali tradizionali.

Nonostante questi rilievi, la scelta di Martini incontrò il favore dei docenti, perché dava loro la possibilità di spiegare agli alunni che il diritto non poteva essere identificato con la norma giuridica e che la norma non doveva essere considerata come la semplice traduzione di principi etici astratti, concepiti al di fuori di un preciso contesto storico e sociale. In un periodo in cui il docente incontrava difficoltà a spiegare le norme del codice pio-benedettino, il saggio di Martini offriva uno spazio maggiore all'approfondimento di tematiche generali.

Toccò a me trattare nella seconda parte il tema della fondazione teologica del diritto canonico ${ }^{15}$. Dovevo svilupparlo adoperando il materiale elaborato nei convegni del Gruppo e tenendo conto del dibattito in atto in quegli anni. Come si è visto, le ipotesi formulate erano diverse e non sempre complementari. Fra gli autori si poneva anzitutto il problema del metodo: quale rilevanza dare all'invito del Vaticano II di tenere presente nella esposizione del diritto canonico il mistero della Chiesa secondo la costituzione dogmatica De Ecclesia? Non meno impegnativo era il problema dei contenuti: a quale nozione di diritto bisognava fare riferimento e come svilupparla? Era sufficiente inserire in una cornice nuova il quadro elaborato dalla dottrina tradizionale? Se si voleva formulare ex novo una diversa ipotesi, era possibile far tesoro delle proposte avanzate nel dibattito in corso in quegli anni?

Non mi fu facile elaborare il testo pubblicato nel primo volume del nostro manuale. Volendo mantenermi fedele alle direttive della Optatam Totius, ritenni doveroso avviare il discorso della fondazione teologica del diritto canonico dalla nozione di «Chiesa-sacramento di salvezza», che troviamo nel primo paragrafo della Lumen Gentium (LG 1, EV 1/284).

Fatta la scelta del quadro ecclesiologico di riferimento, bisognava sviluppare la trattazione in modo coerente. Non ritenevo corretto dal punto di vista metodologico operare una sorta di giustapposizione fra la nuova concezione ecclesiologica e le tradizionali concezioni di di-

is A. Longhitano, Il diritto nella realtà ecclesiale, in Il diritto nel mistero della Chiesa,cit., 39-67. 
ritto, di istituzione, di ordinamento, di norma... Era necessario dimostrare che queste nozioni erano costitutive della Chiesa stessa. La giuridicità non è un elemento accessorio, che si aggiunge alla natura già compiuta della Chiesa, ma una sua dimensione necessaria senza la quale non può esistere.

Il primo tentativo di sintesi non fu molto felice, perché all'interno del Gruppo non si aveva ancora una visione chiara di tutte le tessere del mosaico da comporre. Lo schema che intendevo sviluppare era il seguente: dopo avere analizzato le nozioni teologiche di sacramento e di comunione, mi ripromettevo di illustrare la nozione giuridica di istituzione, per dedurre dalla convergenza di queste diverse nozioni che la Chiesa ha una sua specifica giuridicità, per certi aspetti simile a quella dell'ordinamento statuale e per altri diversa. Decisi di spiegare la nozione di istituzione facendo riferimento a due autori: uno classico (Sinibaldo Fieschi, poi Innocenzo IV), l'altro contemporaneo (Santi Romano). Facendo proprio il pensiero di quest'ultimo, scrivevo che l'istituzione «è un'unità ferma e permanente che non perde la sua identità [...] per il mutarsi dei singoli suoi elementi, delle persone che ne fanno parte, del suo patrimonio, dei suoi mezzi, dei suoi interessi, dei suoi destinatari e così via. Essa può rinnovarsi, conservarsi la medesima, mantenendo la propria individualità». In tal senso l'istituzione poteva essere considerata come «la prima originaria ed essenziale manifestazione del diritto» ${ }^{16}$.

Spiegato il concetto di istituzione, potevo sottolineare nella concezione della Chiesa il nesso esistente fra la categoria teologica del sacramento e la categoria giuridica dell' istituzione per affermare l'esistenza di un ordinamento sostanziale, costituito da una serie di elementi già di per sé strutturati in modo organico: il popolo di Dio radunato dalla parola, i doni dello Spirito, i sacramenti... e di un ordinamento formale, che deve esplicitare l'organizzazione di questi elementi e creare una serie di ruoli, funzioni, modelli di comportamento, norme... L'istituzione per un verso è necessaria e ineludibile, per un altro verso è relativa e funzionale alla natura e alla missione della Chiesa ${ }^{17}$.

Il tentativo di proporre una nuova ipotesi di fondazione del diritto canonico in linea con le indicazioni della Optatam Totius - che

\footnotetext{
${ }^{16} \mathrm{Ivi}, 45-48$.
}

"Ivi, 48-55. 
allo stesso tempo fosse metodologicamente corretta e in grado di recuperare il meglio dell'insegnamento tradizionale - non poteva dirsi del tutto riuscito, perché le nozioni di istituzione e di diritto adoperate non erano formali o neutre, ma risentivano della elaborazione in sistemi di pensiero estranei alla specifica natura della Chiesa.

Bisogna ancora far rilevare che nella prima edizione del nostro manuale il tema della fondazione del diritto canonico veniva ripreso nel capitolo successivo ${ }^{18}$, costituito da due distinte trattazioni: la prima, riportava un saggio di don Gustave Leclerc, pubblicato due anni prima della sua morte, e illustrava le caratteristiche della norma canoni$\mathrm{Ca}^{19}$; la seconda, elaborata da Bruno Senofonte, spiegava gli istituti canonistici peculiari che dovevano aiutare la norma canonica a raggiungere le sue finalità.

Questo secondo capitolo, anche se teoricamente intendeva continuare e sviluppare il discorso fatto in quello precedente, di fatto partiva da ben altre premesse. La concezione di diritto canonico esposta da Leclerc era quella della scuola canơnistica italiana, cioè quella dello Ius publicum ecclesiasticum riformulata dai professori di diritto canonico delle università statali italiane. La Chiesa era considerata una societas perfecta analoga allo Stato; acquistava un carattere soprannaturale a motivo del suo fine e dei mezzi di cui disponeva; la sua giuridicità derivava dal carattere societario, secondo il noto adagio ubi societas ibi ius $^{20}$ e dalla particolare condizione di societas inaequalis, proveniente dal rapporto di subordinazione dei laici ai chierici ${ }^{21}$. Secondo la concezione sottesa negli scritti di questa scuola, il diritto era identi-

${ }^{18} \mathrm{G}$. Leclerc - B. Senofonte, Specificità dell'ordinamento canonico, ivi, 68-115.

${ }^{19} \mathrm{G}$. Leclerc, Configurazione della norma canonica, cit.

${ }^{20}$ «La considerazione del diritto canonico come un elemento derivante necessariamente dalla Chiesa, quale società visibile e gerarchica [...] ci porta ad indagare sulla genesi della norma giuridica nella Chiesa. La nostra asserzione, secondo la quale l'autorità legittima della Chiesa è l'unica fonte prossima o immediata dell'ordine canonico esterno non significa l'esclusione di un apporto attivo e responsabile di tutto il popolo di Dio nell'elaborazione delle norme che compongono l'ordinamento canonico» (Leclerc - Senofonte, Specificità, cit., 75).

${ }^{21}$ «Secondo il disegno divino, una società umana, strutturata gerarchicamente la Chiesa visibile -, acquista un carattere sovrannaturale a motivo del suo fine, la nostra salvezza, e dei mezzi di cui dispone per raggiungerla: la Parola e i sacramenti, fatti veicoli di una grazia elargita attraverso la mediazione di atti esterni, la cui determinazione concreta è affidata alla gerarchia, alla quale compete la potestas sacra di insegnare, santificare e di governare» (ivi, 69). 
ficato con la norma ${ }^{22}$. Si distinguevano norme di diritto divino e norme di diritto positivo. Le norme di diritto divino erano giuridiche perché recepite e promulgate dal legislatore canonico ${ }^{23}$. Nella trattazione non mancavano i riferimenti ai documenti conciliari, anzi si affermava esplicitamente che la Chiesa è sacramento di salvezza; ma si trattava di citazioni estrinseche, che non scalfivano minimamente una concezione di Chiesa e di diritto estranea alla dottrina del Vaticano II.

Altre affermazioni, che potevano prestarsi a qualche ambiguità, erano contenute nel saggio di Attilio Giacobbi ${ }^{24}$. Nella parte introduttiva, illustrando la distinzione fra norme di diritto divino e di diritto positivo, relativizzava di molto la loro differenza e faceva sorgere qualche interrogativo sulla concezione di diritto ad esse sottesa quando affermava che nella Chiesa tutte le norme possono essere considerate di diritto divino, perché le persone che le promulgano «posseggono lo Spirito Santo e operano sotto l'azione dello Spirito» ${ }^{25}$.

Come si può notare da questi riferimenti e da altri — che non è difficile individuare leggendo la prima edizione del manuale - il Gruppo, pur proponendosi di riformulare un nuova ipotesi di rifondazione del diritto canonico secondo le direttive della Optatam Totius, non riusciva ancora ad esprimere una concezione chiara, coerente e unitaria. Era necessario continuare nel cammino intrapreso, fatto di dialogo, di confronti, di incomprensioni per portare a compimento una scelta operata fin dall'inizio della sua formazione.

\subsection{Accoglienza e recensioni del primo volume del manuale}

Con la pubblicazione del primo volume del manuale (1979) il Gruppo uscì allo scoperto ed offrì a tutti la possibilità di valutare

${ }^{22} \mathrm{Nel}$ saggio con l'espressione ,ordinamento canonico" si intende solamente il complesso delle norme giuridiche emanate dall' autorità e come autore di riferimento si cita V. Del Giudice, Nozioni di diritto canonico, edizione rifatta da G. Catalano, Milano 1970. Non era ipotizzabile un diritto come ,ordine necessario" o come ,struttura" derivante dalla natura stessa della Chiesa, che rientrava a pieno titolo nel suo ordinamento giuridico.

${ }^{23}$ «Tanto le norme di diritto divino quanto quelle d'origine umana (Legislatore ecclesiastico) si impongono ai fedeli, quali norme di diritto canonico, in quanto vengono assunte come tali dalla autorità legittima della Chiesa, fonte prossima o immediata dell'ordinamento canonico» (Leclerc - Senofonte, Specificità, cit., 71).

${ }^{24}$ A. Giacobbi, Il diritto nella storia della Chiesa. Sintesi di storia delle fonti e delle istituzioni, in Il diritto nel mistero della Chiesa, cit., 117-236.

${ }^{25} \mathrm{lvi}, 129-130$. 
criticamente le scelte operate sia quanto al metodo sia quanto ai contenuti. Le reazioni furono diverse: la prima che dobbiamo rilevare è quella dei docenti. L'elenco comprende due diverse categorie di persone: a) coloro che frequentano più o meno regolarmente i convegni e conoscono lo stile di lavoro del Gruppo; b) coloro che conoscono il Gruppo solo attraverso i sussidi didattici, il manuale e le circolari.

Chi aveva frequentato i convegni della Mendola conosceva le difficoltà incontrate e l'impegno del Gruppo nella ricerca di un metodo di studio e di insegnamento coerente con le indicazioni del Vaticano II. Il suo giudizio non si fermava ai contributi del manuale o agli schemi di insegnamento, che man mano venivano formulati. La consapevolezza di trovarsi ancora all'inizio di un difficile cammino induceva ad assumere un atteggiamento costruttivo, anche quando i risultati non erano quelli sperati. Diverso era l'atteggiamento di chi, non essendo mai venuto ai convegni, presumeva di formulare un giudizio sul Gruppo e sul suo metodo di studio e di ricerca solo attraverso gli schemi, il manuale o le circolari che gli venivano inviate.

L'accoglienza del manuale da parte dei docenti dei seminari e degli studentati religiosi fu sostanzialmente positiva: pur con i suoi limiti, costituiva un prezioso strumento di lavoro in un momento di estrema difficoltà ad insegnare e a far apprezzare una disciplina, che veniva considerata la causa di tutti i mali della Chiesa ${ }^{26}$.

C'era, poi, la reazione degli addetti ai lavori, che conoscevano l'esistenza del Gruppo ma non avevano ancora avuto modo di valutare il suo operato. Anche questo fronte era alquanto vario: a) per il direttivo dell'Associazione Canonistica - che non aveva mai perdonato al Gruppo di aver organizzato propri convegni annuali in concorrenza con i suoi - i docenti erano elementi pericolosi da tenere d'occhio. Il primo volume del manuale rafforzava questo convincimento dei responsabili dell' Associazione Canonistica e forniva le prove per formulare precisi capi d'accusa verso chi - a loro giudizio - aveva il torto di aver abbandonato i metodi tradizionali di ricerca e di insegnamento per proporre nuove strade ritenute sen-

\footnotetext{
${ }^{26}$ A tal proposito, piace ricordare la testimonianza del prof. Giuseppe Gervasio, durante il convegno della Mendola del 1990: solo seguendo il metodo del vostro manuale sono riuscito a far accettare il diritto canonico ai miei alunni del seminario di Bologna.
} 
za sbocco ${ }^{27}$; b) dalle facoltà di diritto canonico nelle università pontificie giunsero apprezzamenti e incoraggiamenti per il tentativo intrapreso $\left.{ }^{28} ; \mathrm{c}\right)$ i docenti delle università statali si trovavano in profonda crisi e non erano ancora in grado di valutare le diverse proposte di rinnovamento avanzate negli anni del post-concilio.

Meritano una maggiore attenzione le recensioni sul primo volume del manuale apparse nelle riviste. Mi fermo a prendere in esame quella de La Civiltà Cattolica ${ }^{29}$. Il p. Salvatore Lener, che si apprestava a pubblicare una serie di saggi per esporre una propria teoria sulla fondazione del diritto in genere e del diritto canonico in particolare, valutò negativamente la trattazione di Martini sul diritto nella realtà umana, ma apprezzò il mio saggio sul diritto nella realtà ecclesiale. Le motivazioni di questo suo contrastante atteggiamento furono più chiare quando fece conoscere il suo pensiero. Egli aveva del diritto una concezione istituzionale, analoga a quella divulgata da Santi Romano, perciò, notando nel mio saggio che la nozione di istituzione era spiegata con le parole di questo autore, non poteva non manifestare la propria soddisfazione. Tuttavia il giudizio favorevole sul secondo saggio del manuale dava subito l'impressione di essere strumentale a quello negativo del primo di cui non apprezzava né l'impostazione né i contenuti:

«Come bene osserva il prof. Longhitano nella seconda parte, alla base delle polemiche suscitate dagli odierni antigiuridisti giuocano particolari e diverse concezioni (non solo scientifiche) del diritto in genere e persino della Chiesa (p. 56). Sta di fatto, però, che mentre tra gli autentici giuristi si finisce con l'intendersi perché, pur aderendo a indirizzi divergenti (normativisti e istituzionalisti, positivisti e giusnaturalisti), c'è un fondo nucleare di idee comuni o equipollenti (in definitiva, il binomio diritto-giustizia), nelle

\footnotetext{
${ }^{27}$ Ricordo in particolare il discorso fattomi dal presidente dell'Associazione Canonistica, Mons. Angelo Di Felice, dopo aver letto il nostro manuale: a suo giudizio il mio contributo poteva essere considerato accettabile, ma quello di Martini doveva essere eliminato. Per giustificare questo suo giudizio negativo citava il riferimento alle Brigate rosse, indicato dall'autore come esempio dei «centri di potere informale» che in ogni società «giocano un peso notevole» (Il diritto nella realtà umana, cit., 32-33).

${ }_{28}$ Diversi docenti di diritto canonico delle pontificie università facevano parte del nostro Gruppo; fra gli altri è doveroso citare i proff. J.B. Beyer, U. Navarrete e P. Huizing della Gregoriana e il prof. A. Boni dell'Antoniano.

${ }^{29}$ La Civiltà Cattolica 131 (1980) Il, 91-92.
} 
nuovissime concezioni proposte dagli antitradizionalisti si trovano solo costruzioni ideologiche e terminologia seducenti, scarsamente fornite di consistenza e coerenza e, comunque, poco in armonia con l'autentica dottrina cattolica. Spiace di dover fare, sia pure in parte, un rilievo in questo senso alla prima monografia del volume (Il diritto nella realtà umana del prof. A. Martini), quella che pur avrebbe dovuto fornire ai lettori un'idea sicura ed oggettiva, bene utilizzabile nell'intera raccolta, del diritto in genere. Non solo, infatti, se ne propone una nuovissima concezione „come antropologia giuridica”, ma una concezione addirittura „avveniristica”, giacché per l'Autore sulle orme del Garaudy, l'uomo, il diritto e la storia non sono che tre „progetti” o realtà da guardare solo in funzione di un migliore domani $\gg^{30}$.

Per il recensore aveva un particolare significato il riferimento a Roger Garaudy, filosofo marxista, che alcuni anni prima era stato espulso dal Partito comunista francese per le sue idee eterodosse. Invece di giudicare il contenuto del volume citato, tradotto e pubblicato in Italia dalla Cittadella di Assisi ${ }^{31}$, considerava sufficiente un semplice riferimento alla persona, quasi per affermare in chi riferiva il suo pensiero una evidente simpatia per la cultura marxista.

L'attacco del p. Lener continuava facendo rilevare che nel saggio "l'intera costruzione, pur essendo esplicitamente specificata dalla necessità che il „progetto uomo" e il „progetto giuridico" siano ,autenticamente umani e umanizzanti", non appoggia tale necessità su alcun principio assoluto, ma solo su un cosiddetto ,progetto storico”, ch'è quanto dire l'esistenza di un ,nucleo fondamentale di valori condiviso dall'intera umanità" (p. 6 e passim). Ora, questa universale comunanza di valore fondamentali sul piano dei fatti non è mai esistita [...], mentre sul piano dei principi non è affermabile se non nel quadro di quel giusnaturalismo che l'A. non esita affatto a buttare a mare (p. 20)»>32.

Basta un'attenta lettura del primo capitolo del manuale per considerare del tutto infondata la critica formulata dal p. Lener. Il giusnaturalismo buttato a mare era quello illuministico, che prendeva in esame una concezione astratta e razionale dell'uomo, non quello deducibile dal personalismo cristiano. Trattando del „progetto uomo” Martini

\footnotetext{
${ }^{30}$ Ivi., 91 .

${ }^{31}$ R. Garaudy, Progetto speranza, Assisi 1976.

${ }^{32}$ La Civiltà Cattolica 131 (1980) II, 91.
} 
adoperava espressioni chiare che non lasciavano dubbi sulle sue intenzioni:

«A motivo di queste caratterizzazioni funzionali, il progetto di vita non è facoltativo, ma necessario; la sua eventuale assenza ingenera disarmonia interiore e disadattamento sociale. La sua veracità autentica e la sua fecondità è garantita nella misura in cui scaturisce come „vocazione» ${ }^{33}$.

Se poi avesse dato una scorsa alle note, avrebbe potuto costatare che l'autore faceva un continuo riferimento al magistero ecclesiastico (Concilio Vaticano II, encicliche di Giovanni XXIII e di Paolo VI) e che la quasi totalità degli autori citati erano teologi o giuristi cattolici. Perciò non era difficile dedurre che il saggio si muoveva all'interno del personalismo cristiano e il ,quadro di valori” ai quali ancorava il progetto giuridico era quello tradizionale del diritto naturale, considerato però storicamente.

Non mi pare infondato il sospetto che la recensione del p. Lener su La Civiltà Cattolica sia stata "commissionata” da chi voleva scoraggiare il tentativo del Gruppo di proporre un nuovo metodo di ricerca e di insegnamento. Questo stesso espediente fu ripetuto qualche anno dopo per il secondo volume del manuale e per altre pubblicazioni ispirate al nuovo metodo.

Quale che fosse il pensiero dei critici e di coloro che si erano adoperati in vario modo per scoraggiare il Gruppo nel continuare la sua attività, i primi due saggi del nostro manuale sul diritto nella realtà umana e nella realtà ecclesiale, ad insaputa di tutti, dopo la promulgazione del nuovo codice, furono tradotti in castigliano dal prof. Cayetano Rossell C.R. della pontificia università del Messico $^{34}$.

\subsection{La pubblicazione degli altri tre volumi del manuale}

Con la pubblicazione del primo volume del manuale il Gruppo aveva assunto un impegno implicito con i docenti e gli operatori di diritto canonico: offrire a tutti un corso completo di diritto, seguendo gli stessi criteri metodologici del primo volume. La maggior parte dei te-

\footnotetext{
${ }^{3}$ Il diritto nella realtà umana, cit., 6.

${ }^{34}$ Agostino Martini O.F.M. y Adolfo Longhitano, pbro, El derecho en la realidad humana y en la vida de la Iglesia, Colección Teologia del Derecho Canónico, 1987-1988.
} 
mi da inserire negli altri volumi era già stata sviluppata nei convegni annuali ${ }^{35}$. Si trattava, perciò, di organizzare opportunamente la materia. La responsabilità di curare $\mathrm{i}$ tre volumi mancanti fu assegnata a quei docenti che erano allo stesso tempo esperti nei diversi libri del codice e disponibili a coordinare il lavoro con gli autori.

Nel luglio del 1980, a distanza di oltre un anno dalla pubblicazione del primo volume, fu pubblicato il quarto, nel settembre dello stesso anno il terzo e all'inizio del 1981 il secondo. Nella pubblicazione dei quattro volumi del manuale non fu possibile seguire il criterio progressivo per le evidenti difficoltà incontrate nella stesura del secondo volume, destinato a comprendere la trattazione di temi nuovi e impegnativi: il popolo di Dio, i fedeli cristiani, la potestà sacra, la collegialità e il primato, l'ordinamento della Chiesa particolare e della Chiesa universale... Quando la tipografia aveva finito il lavoro di composizione, fu necessario comporre una seconda volta alcuni capitoli sia per tener conto dei nuovi documenti promulgati dai dicasteri romani, sia per meglio armonizzare la trattazione di qualche tema particolarmente difficile.

L'opera, nonostante i suoi limiti, costituì per il Gruppo un risultato di notevole rilievo: era il frutto di un nuovo metodo di studio del diritto canonico, portato avanti collegialmente da un gruppo di docenti e sperimentato concretamente nella scuola; riusciva a trovare un accettabile compromesso per armonizzare la normativa del codice del 1917 con quella post-conciliare; offriva ai docenti un manuale unitario per la scuola, evitando il ricorso a dispense, fotocopie, vciumi non pensati per la scuola o non rispondenti alle esigenze dei seminari.

\subsection{L'inchiesta sul primo volume del manuale e gli apporti al metodo per la fondazione teologica del diritto canonico}

Nel 1981, dopo la pubblicazione di tutto il manuale, il Gruppo decise di avviare un'inchiesta sul primo volume (soprattutto fra

\footnotetext{
${ }^{35}$ Dai temi affrontati nei primi convegni si può stabilire facilmente il cammino seguito: nel secondo anno fu trattato Il popolo di Dio e la sua struttura organica, nel terzo I sacramenti, nel quarto Il rapporto comunità ecclesiale e comunità politica. Questo tema doveva trattarsi nel 1976, ma la morte di don Gustavo Leclerc, indusse il segretario a sospendere per quell'anno il convegno e l'argomento fu trattato nel settembre del 1977. Nel 1978 fu la volta di affrontare il tema della tutela dei diritti e della comunione ecclesiale che comprendeva: i processi, la giustizia amministrativa e la disciplina penale. Nel 1979 si completò il programma con gli schemi sul magistero, sui tempi i luoghi sacri e il culto divino, sul diritto patrimoniale.
} 
i docenti che lo avevano adottato) per avere un loro giudizio sul metodo seguito e sui contenuti, con gli opportuni suggerimenti in vista della seconda edizione.

In un foglio ciclostilato, dopo l'indicazione delle generalità, si chiedeva anzitutto se si conosceva il volume per averlo letto o per averlo adottato. Si ponevano, poi, alcune domande: «Ti piace la sua impostazione o hai delle riserve? Quali? Da quanto tempo lo hai adottato? Quali osservazioni hai da fare sui diversi contributi dal punto di vista dei contenuti, del metodo e della forma? Dovendo provvedere ad una seconda edizione, quali proposte hai da fare? Il progetto del nuovo codice tratta sotto il titolo Norme generali diversi argomenti. Ritieni opportuno trattarli con criterio diverso negli altri volumi secondo la materia?».

Alla segreteria, dentro il 15 novembre 1981, pervennero in risposta 19 fogli da parte di 22 docenti (qualche foglio recava più firme). I rilievi e le osservazioni erano di varia consistenza ma molto interessanti. La maggior parte approvava il metodo seguito e riteneva il volume uno strumento valido per l'insegnamento. Nel giudizio sui singoli contributi i pareri erano divergenti.

Alcuni rilievi critici riguardavano il contributo di Martini: quasi tutti facevano osservare che il linguaggio adoperato era ostico; alcuni lo giudicavano positivamente, perché permetteva agli alunni di collocare il diritto all'interno di un mondo più familiare e condiviso; altri suggerivano di rivederlo e di integrarlo seguendo uno schema più tradizionale; solo alcuni proponevano di eliminarlo del tutto.

Anche sul secondo capitolo erano molti i rilievi e i suggerimenti: alcuni non condividevano l'eccessivo spazio dato alla ecclesiologia; altri suggerivano di integrare meglio la trattazione teologica con quella giuridica per far emergere in modo più chiaro il fondamento ecclesiologico del diritto; altri ancora proponevano uno schema articolato per sviluppare meglio la materia e suggerivano di affidare ad uno solo la trattazione dei due capitoli per avere una esposizione più unitaria ed evitare le ripetizioni. Qualche giudizio più radicale era strettamente legato alla proposta di ridurre i quattro volumi programmati in uno solo; in questo progetto venivano considerati sovrabbondanti, se non inutili, i primi due saggi del primo volume.

Frattanto il dibattito sulla fondazione teologica del diritto canonico si era arricchito con i contributi di diversi membri del Gruppo, che permisero di sciogliere alcuni nodi problematici. Il primo riguardava la correttezza metodologica dell'uso di una nozione formale di diritto 
nel tema della fondazione del diritto canonico. La scuola di Klaus Mörsdorf - Eugenio Corecco in particolare - sosteneva che dal punto di vista metodologico non era corretto usare nel diritto canonico la stessa nozione di diritto adoperata per gli ordinamenti civili, perché si rischiava di introdurre elementi estranei che avrebbero finito col falsare le conclusioni. Il diritto canonico aveva il suo fondamento negli elementi che costituiscono la Chiesa: la parola e il sacramento. Il carattere giuridico vincolante della parola sta nel fatto che essa avviene in nome e per mandato di Cristo. Il carattere giuridico del sacramento consiste nella sua possibilità, come simbolo, di esprimere o di realizzare le obbligazioni giuridiche sotto forma di figura.

Per i docenti, che condividevano la preoccupazione di seguire il metodo teologico nel formulare una ipotesi di fondazione del diritto canonico, non era facile capire e spiegare questa dottrina. Dal punto di vista didattico, prima di affrontare il problema, il docente doveva procedere ad una sorta di explicatio terminorum. Se si voleva seguire l'ipotesi della scuola di Mörsdorf i termini da spiegare erano diversi: non solo il termine „diritto”, ma añche „parola” e „,sacramento” per capire in che senso costituiscono la Chiesa e perché mai si dovesse loro riconoscere una valenza giuridica. Ma gli autori non andavano oltre le semplici affermazioni generali e lasciavano ai docenti il difficile compito di interpretare il loro pensiero.

Due apporti contribuirono a chiarire l'ipotesi di fondazione del diritto canonico formulata nel primo volume del manuale: alcuni scritti di Francesco Coccopalmerio ${ }^{36}$ e uno di Velasio De Paolis ${ }^{37}$, che si erano già inseriti nel Gruppo e frequentavano assiduamente i convegni. Il primo faceva notare che il ricorso ad una nozione di diritto per affrontare il tema della fondazione del diritto canonico, non solo non era metodologicamente scorretto ma doveva essere considerato necessario, perché la teologia non può fare a meno di adoperare il linguaggio umano; l'unica avvertenza era di far ricorso ad una nozione formale, cioè ad una nozione estremamente povera di significato, purificata da ogni elemento estraneo proveniente dalle concezioni delle diverse scuole. De Paolis scriveva che non è corretto interrogarsi se la nozio-

${ }^{36}$ F. Coccopalmerio, De conceptu et natura iuris Ecclesice animadversiones quaedam, in Periodica 66 (1977) 447-497; Id., Fondare teologicamente il diritto nella Chiesa?, in La teologia italiana oggi, Brescia 1979, 395-410.

${ }^{37}$ V. De Paolis, Ius: notio univoca an analoga? in Periodica 69 (1980) 127-161. 
ne di diritto da applicare alla realtà ecclesiale deve essere uguale o diversa da quella adoperata nella realtà umana, perché le nozioni formali sono uguali per tutti.

Gli apporti a un metodo teologico per la fondazione del diritto canonico non giunsero solo dai membri del Gruppo. Tanti altri canonisti di diversa provenienza si erano già posti gli stessi problemi e avevano avanzato proposte analoghe. Si pensi alle opere di Javier Hervada, Pedro Lombardia e Pedro Juan Viladrich, ai saggi di teologi e canonisti delle università di Strasburgo, Parigi e Salamanca, agli scritti di Remigius Sobanski... Anche nelle università italiane incominciava a formarsi una nuova generazione di canonisti, che avevano abbandonato la dottrina dei loro maestri per far proprie le indicazioni del Vaticano II.

\subsection{La promulgazione del nuovo „Codice di diritto canonico" e il convegno del 1983}

L'ipotesi del Gruppo sulla fondazione del diritto canonico sembrava ormai avviarsi ad un definitivo chiarimento, quando fu annunziata la promulgazione del nuovo Codice di diritto canonico. Il discorso fatto dal Papa in occasione della presentazione del Codice ${ }^{38}$ e la costituzione apostolica Sacrae Disciplinae Leges ${ }^{39}$ furono per tutti una piacevole sorpresa, perché in questi documenti si aveva una conferma indiretta della correttezza del metodo seguito dal Gruppo fin dal suo costituirsi $i^{40}$.

Nella sua costituzione Giovanni Paolo II distingueva chiaramente fra diritto e legge ${ }^{41}$; considerava il diritto connaturale alla Chiesa stes-

${ }^{38}$ Discorso di Sua Santità Giovanni Paolo II, in Communicationes 15 (1983) 9-16.

${ }^{39}$ EV 8/611-648. Per un commento alla costituzione apostolica vedi: G. Ruggieri, L'ecclesiologia conciliare nelle strettoie giuridiche, in Il Regno-Attualità 128 (1983) $6,143-146$.

${ }^{40}$ Il tema della fondazione teologica del diritto canonico, con riferimento ai documenti della promulgazione del codice, è affrontato nel saggio di A. Longhitano, Chiesa, diritto e legge nella costituzione apostolica "Sacrae Disciplinae Leges”, in ME 108 (1983), 399-435 e in A venti anni dal concilio. Prospettive teologiche $e$ giuridiche. Atti del Convegno di studi "Il Concilio Vaticano II venti anno dopo". Catania 21-22 Aprile, 5-6 maggio 1983, Palermo 1984, 109-140.

${ }^{41} \ll$ Se la Chiesa-corpo di Cristo è compagine organizzata, se comprende in sé detta diversità di membra e di funzioni, se ,si riproduce” nella molteplicità delle Chiese particolari, allora tanto fitta è in essa la trama delle relazioni che il diritto c'è già, non può non esserci. Parlo del diritto inteso nella sua globalità ed essenzialità, prima ancora delle specificazioni, derivazioni o applicazioni di ordine puramente canonico [...]. Connaturale è il diritto alla vita della Chiesa» (Giovanni Paolo II, Discorso, cit., 14). 
sa, ma allo stesso tempo faceva rilevare l'importanza della legge perché la Chiesa potesse svolgere la sua missione di salvezza ${ }^{42}$. Particolarmente significativo appariva il confronto fra l'ecclesiologia del codice e quella del Vaticano II con conseguente criterio ermeneutico di far riferimento ai documenti del concilio nel caso in cui il linguaggio canonistico non fosse riuscito a tradurre perfettamente l'immagine della Chiesa:

«Lo strumento, che è il codice, corrisponde in pieno alla natura della chiesa, specialmente come vien proposta dal magistero del concilio Vaticano II in genere, e in particolar modo dalla sua dottrina ecclesiologica. Anzi, in un certo senso, questo nuovo codice potrebbe intendersi come un grande sforzo di tradurre in linguaggio canonistico questa stessa dottrina, cioè la ecclesiologia conciliare. Se poi è impossibile tradurre perfettamente in linguaggio „canonistico” l' ,immagine" della Chiesa, tuttavia a questa immagine il codice deve sempre riferirsi, come a esempio primario, i cui lineamenti esso deve esprimere in se stesso, per quanto è possibile, per sua natura» (EV 8/628).

Queste parole non potevano non-richiamare alla mente le polemiche sorte negli anni precedenti dentro e fuori il Gruppo sulla scelta di avere un costante riferimento alla dottrina del Vaticano II per affrontare il tema della fondazione del diritto canonico e per una corretta comprensione degli istituti e delle norme del codice. Nessuno certamente si sarebbe aspettato una così autorevole conferma.

Nel 1982 era stato scelto come tema del convegno annuale del Gruppo la fondazione del diritto canonico e si era pensato di invitare esponenti di altre scuole per un confronto aperto. La promulgazione del nuovo Codice di diritto canonico nel gennaio 1983 ne confermò l'opportunità. Vista l'importanza dell'argomento, si pensò di dedicarvi due giorni interi: al mattino del lunedì era prevista una relazione storico-introduttiva di Eugenio Corecco dal titolo: Per una fondazione

\footnotetext{
$\$ 2$ «Il codice, dal momento che è il principale documento legislativo della Chiesa, fondato nell'eredità giuridico-legislativa della rivelazione e della tradizione, va riguardato come lo strumento indispensabile per assicurare il debito ordine sia nella vita individuale e sociale, sia nell'attività stessa della chiesa. Perciò, oltre a contenere gli elementi fondamentali della struttura gerarchica e organica della chiesa quali furono stabiliti dal suo divin Fondatore oppure radicati nella tradizione apostolica, $o$ in ogni caso antichissima, ed oltre alle principali norme concernenti l'esercizio del triplice ufficio affidato alla stessa chiesa, il codice deve definire anche alcune regole e norme di comportamento» (EV 8/627).
} 
del diritto canonico: il problema alla vigilia del Concilio Vaticano II. Nel pomeriggio il tema sotto il profilo dell'attualità - soprattutto alla luce dei documenti di promulgazione del nuovo codice - era affrontato da Piero Antonio Bonnet, Francesco Coccopalmerio, Eugenio Corecco e Velasio De Paolis in una tavola rotonda dal titolo Per una fondazione del diritto canonico.

Agli interventi dei relatori seguì la discussione molto più vivace e partecipata del solito, nella quale si notò che fra i presenti c'era una sostanziale convergenza. Lo stesso Corecco riconobbe che prima di affrontare il tema della fondazione del diritto canonico era necessario intendersi su una nozione elementare di diritto, che avrebbe potuto essere concepito come „ordine necessario". Allo stesso tempo disse che era nelle sue intenzioni affrontare al più presto in un saggio questo argomento.

A conclusione dei lavori, i relatori decisero di formulare alcune proposizioni di sintesi del loro pensiero per aiutare i docenti a trarne le opportune conseguenze per l'insegnamento.

«1. Nella Chiesa esiste una realtà chiamata ,giuridico-ecclesiale". Bisogna ricercare in modo rigoroso il concetto di questa realtà. Sembra che l'unico luogo per trovare questo concetto sia l'autocoscienza della Chiesa.

2. L'autocoscienza della Chiesa o ci fornisce un concetto di giuridico-ecclesiale elaborato in modo diretto e immediato, oppure assume il concetto di giuridico dalla esperienza umana e lo applica a sé. I relatori sono del parere che la Chiesa abbia assunto il concetto di giuridico dalla esperienza umana e lo abbia applicato a se stessa.

3. I relatori concordano sostanzialmente su un concetto di diritto che si fonda sulla persona in quanto è in relazione necessaria e ordinata nella comunità.

4. Questo concetto di provenienza umana nell'applicazione alla Chiesa si carica dei contenuti specifici della Comunità ecclesiale. In questo contesto la persona è il christifidelis, la comunità è la Chiesa e le relazioni sono i rapporti in ordine alla salvezza. Il contenuto è nel diritto divino e nella norma ecclesiale in quanto attualizza il diritto divino.

5. Benché in linea di principio si affermi la distinzione tra morale e diritto, nel contesto particolare della Chiesa può risultare difficile e al limite impossibile determinarne i confini precisi».

Le proposizioni redatte dai relatori, i rilievi emersi nel dibattito del convegno e le osservazioni fatte dai docenti nell'inchiesta avviata due anni prima, costituirono i criteri di revisione del primo volume del no- 
stro manuale, la cui seconda edizione fu pubblicata nel 1986. Martini, pur non abbandonando lo schema dell'antropologia giuridica, rinnovò profondamente il suo contributo nei contenuti e nel linguaggio. Nella seconda parte, avviai la trattazione spiegando la nozione di Chiesa-sacramento, ma - secondo le indicazioni ricevute - per spiegare il giuridico-ecclesiale ho adoperato alcune nozioni formali, illustrate nel loro significato etimologico, senza riferimenti a dottrine filosofiche o teologiche: il diritto come istituzione, come struttura, come ordine di giustizia, come hominis ad hominem proportio. Ritenni quanto mai opportuno accennare brevemente al problema del metodo, per far comprendere le motivazioni che avevano spinto il Gruppo a distaccarsi dalle ipotesi formulate dalla canonistica preconciliare. Mantenni l'appendice con l'esposizione delle teorie sulla fondazione del diritto canonico e sviluppai ex novo il capitolo sull'ordinamento positivo.

La promulgazione del codice obbligò il Gruppo a preparare la seconda edizione di tutto il manuale, che si decise di ridurre a tre volumi. Non si trattò di un semplice lavoro di aggiornamento ma di una completa rielaborazione dell'opera, eonsiderato che ormai esisteva un testo legislativo al quale bisognava fare necessariamente riferimento. Nel 1990 fu pübblicato il secondo volume e nel 1992 il terzo.

\section{Le altre iniziative del Gruppo}

I convegni erano stati per il Gruppo il momento di maggiore aggregazione e di più fecondo lavoro. Dopo la promulgazione del codice fu necessario iniziare una nuova fase dei convegni: la prima aveva avuto come oggetto la preparazione del programma e degli schemi d'insegnamento, la seconda era stata dedicata ai lavori della Commissione e alla trattazione di alcuni temi generali, la terza doveva prendere in esame il nuovo codice. Si noti il sottotitolo che si legge nei programmi degli anni 1984, 1985, 1986: «Studio approfondito del Codice di diritto canonico».

Con il convegno di Torino del 1998 si chiuse l'esame dei diversi libri del codice e si decise di passare ai convegni tematici: un tema di attualità e particolarmente rilevante viene sviluppato tenendo conto delle problematiche teologiche e storiche di fondo e con riferimento ai diversi libri del codice.

Dal 1982 il Gruppo è riuscito a realizzare il progetto a lungo vagheggiato di pubblicare gli atti dei convegni, che hanno avuto una positiva accoglienza e costituiscono ormai un punto sicuro di riferimen- 
to per la dottrina, la scuola e la prassi. Il titolo della collana Quaderni della Mendola - ricorda ai membri più anziani il luogo, per tanti aspetti mitico, in cui il Gruppo trovò i suoi momenti di maggiore vitalità e coesione ${ }^{43}$.

Il Gruppo fin dai primi anni della sua formazione aveva preso un'altra iniziativa: la compilazione di elenchi bibliografici sui volumi e sui saggi che man mano venivano pubblicati. Dopo la promulgazione del codice si pensò di fare un lavoro più completo coinvolgendo diversi collaboratori. Il progetto preparato e coordinato da don Carlo Redaelli prevedeva la schedatura dei volumi di diritto canonico e dei saggi, apparsi nelle riviste specializzate e non, a partire dalla promulgazione del codice.

La realizzazione di questa iniziativa si rivelò particolarmente difficile, perché erano previste diverse fasi: nella prima gli incaricati dovevano compilare le schede secondo i criteri stabiliti; nella seconda don Carlo Redaelli e l'équipe dei collaboratori dovevano rivedere il materiale ricevuto e trascriverlo per poi passarlo alla tipografia. Il Centro Ambrosiano di documentazione e di studi religiosi aveva dato la propria disponibilità alla pubblicazione. Una prima quota di schede fu pubblicata nel 1988, ma si pose subito il problema delle tecniche più idonee per continuare il lavoro: invece di adoperare le schede di cartone si pensava di passare ai dischetti del computer. Fu necessario individuare una ditta specializzata a cui affidare il compito di preparare un programma da utilizzare nelle diverse fasi della preparazione, della revisione e della utilizzazione delle schede. Il primo tentativo non giunse a buon fine e fu necessario cercare tecnici più validi. Intanto, ritenendo senza prospettive la via della commercializzazione di questa iniziativa, si decise di collocare le schede nella rete di internet per consentire a tutti la consultazione gratuita. Oggi chiunque può connettersi al sito del Gruppo www.giddc.org e consultare liberamente le schede bibliografiche, che i responsabili hanno preparato e che man mano si impegnano ad aggiornare.

\section{Conclusione}

Sono due $\mathrm{i}$ criteri di lavoro e le caratteristiche principali che hanno guidato il Gruppo italiano docenti di diritto canonico nella sua attività

${ }^{43}$ La collana oggi (giugno 2000) consta di otto volumi. 
trentennale: uno stile di collegialità e di fraternità, la coerenza nella scelta metodologica iniziale. Fin dall'inizio il Gruppo volle che i lavori si svolgessero ponendo tutti sullo stesso piano e coinvolgendo i membri nelle decisioni. Man mano che si è andati avanti e il Gruppo ha acquisito nuovi membri si è cercato di offrire a tutti pari opportunità, seguendo il principio della competenza e del giusto ricambio. Considerato il metodo seguito nella elaborazione dei programmi e degli schemi, si può affermare a ragione che il soggetto responsabile delle diverse iniziative attuate (i convegni, il manuale, le schede bibliografiche) è il Gruppo. La collegialità nel metodo di lavoro è stata sempre vissuta in un clima di autentica fraternità. Coloro che per la prima volta hanno partecipato ai convegni, hanno subito notato che non c'era l'atmosfera formale ed accademica che di solito si trova in incontri analoghi.

La coerenza alla scelta metodologica iniziale è costata non poche sofferenze e l'autoallontanamento nel corso degli anni di chi non la condivideva. Nonostante le difficoltà incontrate, bisogna riconoscere che alla fine è risultata una scelta vincente. Il Gruppo docenti può attribuirsi il merito di avere elaborato e attuato un metodo che, dopo il concilio, ha permesso di contrastare la ventata antigiuridica e, negli anni successivi, ha consentito di far risaltare nel diritto canonico la fondamentale dimensione di disciplina teologica senza fargli perdere la sua specificità giuridica.

$\grave{E}$ ovvio che il metodo proposto inizialmente, nel corso degli anni, ha subito aggiustamenti e modifiche. Quando fu elaborato poteva anche trovare giustificazione il rilievo di una eccessiva attenzione ai problemi teologici. Infatti subito dopo il concilio anche per i canonisti era preminente l'interesse a riscoprire la rivoluzione copernicana operata nella ecclesiologia e nei sacramenti. Inoltre le incertezze sulla vigenza delle norme del codice pio-benedettino inducevano a sottolineare l'aspetto teologico a scapito di quello normativo. Dopo la promulgazione del Codice di diritto canonico è stato inevitabile ristabilire un certo equilibrio: non veniva meno il riferimento ai principi teologici - che oltretutto il legislatore aveva ritenuto necessario sintetizzare nei canoni introduttivi delle diverse tematiche giuridiche - ma l'attenzione principale è stata rivolta alle norme vigenti.

Queste brevi note sulla storia del Gruppo, più che un carattere celebrativo, intendono avere il significato di una testimonianza, perché si conservi la memoria di una esperienza che non può considerarsi conclusa. 